\title{
Preparation of thermoresponsive core-shell copolymer latex with potential use in drug targeting
}

\author{
Chia-Lung Lin, Wen-Yen Chiu *, Chia-Fen Lee ${ }^{1}$ \\ Department of Materials Science and Engineering, National Taiwan University, Taipei, Taiwan, Republic of China
}

Received 10 March 2005; accepted 19 April 2005

Available online 8 June 2005

\begin{abstract}
A core-shell copolymer latex with thermal-responsive properties was prepared and its potential application as a vehicle for drug targeting was investigated in this work, where the crosslinked copolymer of $N$-isopropylacrylamide (NIPAAm) and chitosan was prepared as the core and the copolymer of methacrylic acid (MAA) and methyl methacrylate (MMA) was prepared as the shell. By using soapless dispersion polymerization, the poly(NIPAAm-chitosan) crosslinked copolymer latex was synthesized first. Then the monomers of MAA and MMA were added to continue the reaction to obtain the core-shell copolymer latex. The weight ratio of MAA/MMA and the concentration of shell monomers (MAA and MMA) in the feed of the reaction mixture had been changed to investigate their effects on the particle size, reaction rate, zeta-potential, specific surface area, and surface functional groups of the latex particles. The swelling and thermoresponsive behavior of the film made from these core-shell latices were also studied under different $\mathrm{pH}$ values of buffer solution. The model drug (caffeine) could be merged inside the copolymer particles and protected from releasing through the transport process effectively. And the thermoresponsive property of these copolymer particles significantly enhances the ligand (protein) conjugation that shows the potential of the latex being applied on the targeting drug carrier.
\end{abstract}

(C) 2005 Elsevier Inc. All rights reserved.

Keywords: Poly(chitosan-NIPAAm/MAA-MMA); Core-shell copolymer latex; Shell composition-swelling-drug release relationship; Thermoresponsive

\section{Introduction}

Thermoresponsive hydrogels are known to exhibit phase separation properties in aqueous solution when the temperature is increased above a certain level. This phase separation temperature is referred to as the lower critical solution temperature (LCST). One such material is the crosslinked $\operatorname{poly}(N$-isopropylacrylamide) (PNIPAAm), and its behavior of temperature-responsiveness has been well documented [1-3]. Recently, PNIPAAm hydrogels are attracting more and more interest in biomedical applications [4-7], because they exhibit a well-defined LCST in water at around 31$34^{\circ} \mathrm{C}$, which is close to the body temperature. By find-

\footnotetext{
* Corresponding author. Fax: +886223623259.

E-mail address: ycchiu@ccms.ntu.edu.tw (W.-Y. Chiu).

1 Present address: Department of Cosmetic Science, Chia Nan University of Pharmacy and Science, Tainan, Taiwan, Republic of China.
}

ing out the right balance of hydrophobic and hydrophilic comonomers and by adjusting the number of electric charges in the chain as well as the degree of crosslinking, the structure and physical properties of PNIPAAm hydrogels can be changed [8-14]. Hence, these gels can be expected to act as intelligent materials used in many fields, such as in controlled or targeted drug release [9,15-18]. A self-regulated drug delivery system has been of great interest because it allows drugs to be released when it is needed.

Drug release from the homopolymeric and copolymeric PNIPAAm gels is affected not only by temperature but also by the dimensions of the gel, the hydrophobicity of the polymer, and the topological structure of the network, as well as by $\mathrm{pH}$. By increasing the thickness of the gel membrane, the drug release rate decreases. Lim et al. [19] have studied the release of indomethacine from PNIPAAm/polyurethane IPNs. Their studies showed that with increasing the hydrophobicity of the gel, the equilibrium drug release and 
the time to reach the equilibrium decreased because of the decrease of the drug loading and the equilibrium swelling ratio. The release rate of indomethacin [20] as well as that of sodium dodecyl sulfate (SDS) [21] from hydrophobically modified PNIPAAm gels was shown to decrease because of the hydrophobic interactions between drugs and the network chains. When acrylic acid was introduced into the PNIPAAm network [22], it was observed that the release of indomethacin occurred at a much higher rate at $\mathrm{pH} 7.4$ than at $\mathrm{pH}$ 1.4. Yoo et al. [23] studied the influence of polyelectrolyte [poly(allyl amine)] on the LCST of $\operatorname{poly}(N$ isopropylacrylamide-co-acrylic acid) [poly(NIPAAm-coAA)] hydrogels. The LCST of the poly(NIPAAm-co-AA) hydrogels in the presence of poly(allyl amine) could be observed even above the $\mathrm{p} K_{a}$ value of PAA. Kim et al. [24] prepared the graft copolymer or blending of chitosan and PNIPAAm. They pointed out that in the buffer solution of various $\mathrm{pH}$ values and temperatures, the chitosan/PNIPAAm blend IPN had a somewhat higher swelling than that of the chitosan-g-PNIPAAm IPN. Lee and Chen [25] have studied the preparation and swelling behaviors of the chitosan/PNIPAAm semi-IPN and IPN hydrogels. They synthesized a new kind of full-IPN chitosan/PNIPAAm hydrogel, which was prepared by chemical combination of methylene bis-acrylamide cross-linked PNIPAAm network with formaldehyde cross-linked chitosan network.

In our previous study [26,27], the poly(chitosanNIPAAm) copolymer particles via soapless dispersion copolymerization [26-28] was designed for drug delivery systems in order to have the advantage of temperature sensitivity and biodegradability. But it was found that when the swollen poly(chitosan-NIPAAm) copolymer particles were put into a $37^{\circ} \mathrm{C}$ buffer solution from room temperature suddenly, the drug solution trapped in it would be released quickly. In order to increase the amount of drug trapped inside the latex particles during the transport process in the human body, a novel poly(chitosan-NIPAAm/MAA-MMA) coreshell copolymer latex was designed and prepared. The added MAA-MMA shell part could protect the drug from releasing and provide the carboxylic acid groups for the conjugation of ligand (protein). The residual reactants after the core synthesis, chitosan and NIPAAm, were incorporated into the shell region and formed "channels" which were expected to let the enzyme (lysozyme) pass through the shell to degrade chitosan in the core. Therefore, these latices could be applied in the drug targeting that protect the drug from releasing through the transport process in human body and then target wounded tissue. Once the ligand targets the wounded cells and causes endocytosis, the drug trapped inside the latex particles would be released after the regulative degradation of copolymer particles in lysosomes inside the cells. The shell composition of the latex was varied, and the effect of it on the morphology and swelling of the core-shell particles was discussed. The thermosensitive and drug-release behavior of the samples with different shell compositions were also studied under different $\mathrm{pH}$ values of buffer solution.
Table 1

Ingredients and conditions for synthesis of crosslinked poly(chitosanNIPAAm/MAA-MMA) copolymer latex $\left(\mathrm{N}_{2} ; 50^{\circ} \mathrm{C}\right.$; stirring rate, $\left.300 \mathrm{rpm}\right)$

\begin{tabular}{llll}
\hline First stage (core latex) & & \multicolumn{2}{l}{ Second stage (core-shell latex) } \\
\hline NIPAAm (g) & 7 & MAA (g) & $0.35-2.1$ \\
Chitosan (g) & 1.4 & MMA (g) & $1.75-7$ \\
MBA (crosslinking agent) (g) & 0.21 & Reaction time (h) & 1 \\
Initiator (APS) (g) & 0.7 & & \\
Acetic acid (g) & 1.5 & & \\
Deionized water (g) & 280 & & \\
Reaction time (h) & 2 & & \\
\hline
\end{tabular}

\section{Experimental}

\subsection{Materials}

$N$-isopropylacrylamide (NIPAAm) was distilled under a nitrogen atmosphere and reduced pressure prior to polymerization. Water was redistilled and deionized. The chitosan, methacrylic acid (MAA), methyl methacrylate (MMA), initiator (ammonium persulfate (APS)), crosslinking agent ( $N, N^{\prime}$-methylenebisacrylamide (MBA)), bovine serum albumin (BSA, $M_{\mathrm{w}} \sim 66 \mathrm{kDa}$ ), and 1-ethyl-3-(3-dimethylaminopropyl) carbodiimide (EDC) were purchased from the Sigma Chemical Company and used as received.

\subsection{Soapless dispersion copolymerization}

The crosslinked poly(chitosan-NIPAAm/MAA-MMA) core-shell copolymer latex was synthesized by the method of soapless dispersion copolymerization [26-28]. In the first stage of this copolymerization, the core latex, poly (chitosanNIPAAm), was prepared as mentioned in our previous studies $[26,27]$ under the conditions listed in Table 1 . The polymerization was carried out for $2 \mathrm{~h}$ at $50^{\circ} \mathrm{C}$ in a nitrogen atmosphere with a $300 \mathrm{rpm}$ stirring rate. After the first stage of synthesis, the second stage of copolymerization was followed under the condition listed in Table 1. Finally, the core-shell latex particles with poly(chitosan-NIPAAm) as core and poly(MAA-MMA) as shell were synthesized. Then the latex product underwent dialysis for 7 days in order to remove unreacted components. The sample, for example, with the code of $2 / 10-3 \%-1 / 5$, had a feed composition of $2 / 10$ in the weight ratio of chitosan/NIPAAm, $3 \%$ in the concentration of crosslinking agent in MBA (g)/NIPAAm (g), and 1/5 in the weight ratio of MAA $(\mathrm{g}) / \mathrm{MMA}(\mathrm{g})$.

\subsection{Conversion}

After the copolymerization, a sample of the latex solution was taken out of the reactor. A quantitative amount of inhibitor was introduced into the latex sample, and the sample was cooled to room temperature for more than $1 \mathrm{~h}$, and then reheated to $60^{\circ} \mathrm{C}$ (above the LCST of PNIPAAm) to obtain the precipitates of the copolymers. For the removal of the remaining monomer and initiator, the copolymer was 
washed with hot water several times and then dried in a vacuum oven at $50^{\circ} \mathrm{C}$ until the weight was no longer changed. The conversion of the monomers was calculated as

conversion $=P /\left(W M_{0}\right) \times 100 \%$,

where $P$ is the weight of the dry copolymer obtained from the latex sample, $W$ is the weight of the latex sample taken out from the reactor, and $M_{0}$ is the weight fraction of monomers in feed.

\subsection{Morphology of copolymer particles}

To observe the morphology of the copolymer latex, the latex solution was coated on a glass plate and observed by a TEOL JSM-6300 scanning electron microscope (SEM). In addition, the samples were microtomed to form sections about $900 \AA$ thick. The ultrathin cross sections were stained with $1 \%$ uranyl acetate solution for $2 \mathrm{~min}$. Warm distilled water was used for the final rinse of the grid to avoid uranyl acetate precipitate. The stained ultrathin cross sections were observed by using a transmission electron microscope (Hitachi H-600).

\subsection{Measurement of swelling ratio}

Since the latex particles were too small for their equilibrium swelling ratio to be measured, the latex particles were dried and modeled to form a particle-formed film in an oven under a temperature of $50^{\circ} \mathrm{C}$ to measure the equilibrium swelling ratio (not the swelling kinetics). The film was immersed in an excess of deionized water for $30 \mathrm{~min}$ to remove the residual unreacted compounds. The swollen polymer film was then cut into disk forms $8 \mathrm{~mm}$ in diameter and dried at $50^{\circ} \mathrm{C}$ until the weight was no longer changed. A preweighed dried sample (Wd) was immersed in an excess of the $\mathrm{pH} 4,7.4$, and 9 phosphate buffer solution in a thermostatic water bath until the swelling equilibrium was attained (i.e., the weight of the swollen film was no longer changed). The weight of the wet sample $\left(W_{\mathrm{s}}\right)$ was determined after the surface water was removed by blotting with filter paper. The equilibrium-swelling ratio (SR) was calculated from the following formula:

$\mathrm{SR}=\left(W_{\mathrm{s}}-W_{\mathrm{d}}\right) / W_{\mathrm{d}}$.

Although the shapes and forms of the samples will influence the swelling kinetics, those factors will not change the equilibrium swelling data.

\subsection{Zeta potential and light scattering measurements}

The zeta potential and hydrodynamic diameter (diameter of swollen copolymer particles) were measured using laser light scattering (Malvern Zetasizer 3000 ) at $25^{\circ} \mathrm{C}$ where the samples had been dialyzed for 7 days and diluted with various phosphate buffer solutions of $\mathrm{pH} 4,7.4$, and 9 .

\subsection{Specific surface area and surface functional group analysis}

The latex of copolymer particles was dialyzed for 7 days and freeze-dried to get the powder to analyze the specific surface area and the amount of carboxylic acid groups on the surface of copolymer particles. The BET specific surface area of particles was measured and analyzed using a Micromeritics ASAP-2100. A given quantity of the powder of copolymer particles was added into deionic water, followed by ultrasonification for $5 \mathrm{~min}$. The number of carboxylic acid groups on the surface of copolymer particles was estimated by $0.01 \mathrm{~N} \mathrm{NaOH}$ titration using conductivity measurements.

\subsection{Caffeine release experiment}

Preweighed dried copolymer particles $(0.15 \mathrm{~g})$ were immersed in a $\mathrm{pH} 7.4$ phosphate buffer solution containing caffeine $(0.012 \mathrm{~g}$ caffeine $/ 5 \mathrm{~g} \mathrm{pH} 7.4$ phosphate buffer solution), followed by ultrasonification for $10 \mathrm{~min}$ and held at a temperature of $4{ }^{\circ} \mathrm{C}$ for $3 \mathrm{~h}$ to attain the swelling equilibrium. Then, these solutions were freeze-dried to obtain the caffeine incorporated copoplymer powder. $0.05 \mathrm{~g}$ of this powder was placed on a mold and pressed with 9 tons of force, and the caffeine-incorporated copoplymer disk with diameter $1 \mathrm{~cm}$ was prepared. The weight of the caffeine-incorporated copolymer disk was designated as $W_{\mathrm{c}}$. Therefore, the caffeine loaded into the disk could be calculated as follows:

$L_{\mathrm{c}}(\mathrm{g}$ caffeine loaded into disk $)=W_{\mathrm{c}} \frac{0.012}{0.15+0.012}$.

The caffeine-incorporated dry copolymer disk was immersed into a phosphate buffer solution $\left(100 \mathrm{~g}, 37^{\circ} \mathrm{C}\right.$, $\mathrm{pH}$ 7.4) to release the caffeine. The quantity of caffeine released from each sample into buffer solution $\left(R_{\mathrm{c}}\right)$ was measured using a UV-vis spectrophotometer (Thermo Spectronic gamma series) by detecting the characteristic absorbance of caffeine at $272 \mathrm{~nm}$. The release percent of caffeine was determined as follows:

release percent $(\%)=R_{\mathrm{c}} / L_{\mathrm{c}} \times 100 \%$.

\subsection{Protein conjugation}

Preweighed dried copolymer particles $(0.01 \mathrm{~g})$ were immersed in a $\mathrm{pH} 7.4$ phosphate buffer solution $(10 \mathrm{~g})$, followed by ultrasonification for $10 \mathrm{~min}$ to suspend the copolymer particles in the solution homogeneously. Then, $0.004 \mathrm{~g}$ BSA was added into the solution and followed by reultrasonification for $3 \mathrm{~min}$. After the solution was placed in a 25 or $37^{\circ} \mathrm{C}$ thermostatic environment for $2 \mathrm{~h}, 0.004 \mathrm{~g}$ EDC was added into the solution for $4 \mathrm{~h}$ to conjugate BSA on the surface of copolymer particles. For estimating the amount of BSA conjugated on the surface of copolymer particles, the solution was centrifuged for $20 \mathrm{~min}$ at $12,000 \mathrm{rpm}$. The quantities of BSA remaining in the solution, which were not 
conjugated on the particles, was measured using a UV-vis spectrophotometer (Thermo Spectronic gamma series) by detecting the characteristic absorbance of BSA at $280 \mathrm{~nm}$.

\section{Results and discussion}

\subsection{Conversion of copolymerization}

The synthesis of the core-shell copolymer particles was conducted by a two-stage process. In the first stage, the poly(chitosan-NIPAAm) core particles were prepared by soapless dispersion polymerization for $2 \mathrm{~h}$. Thermal decomposition of the ionic initiator $\left(\mathrm{S}_{2} \mathrm{O}_{8}^{2-}\right)$ initiated the free-radical polymerization. Initially, NIPAAm was a watersoluble monomer, and oligomers of chitosan-NIPAAm formed in water. As the reaction proceeded, when the crosslinking degree achieved a certain level and the length of the oligomers exceeded the solubility limit, poly(chitosanNIPAAm) precipitated out of the water phase and formed the primary particles. The primary particles underwent aggregation, thereby increasing the surface charge, until electrostatic stabilization was achieved. The particles grew with the aggregation and became more hydrophobic when polymerization was carried out at a temperature higher than LCST. This process resulted in a decrease in the absorption of the hydrophilic oligomers or monomers into the particles. So the particle size of poly(chitosan-NIPAAm) observed by TEM during the polymerization did not increase significantly (mentioned in our previous study [26]). The polymerization of the remaining monomers and oligomers was continued, achieving the critical value required for particle formation, and the particle concentration increased with the reaction time.

After the first-stage reaction, the monomers of MAA and MMA were added into the reaction latex to synthesize the shell part of the particles. The second-stage reaction was similar to the seeded emulsion polymerization, in which the poly(chitosan-NIPAAm) particles synthesized in the firststage one were the seeds. The MAA and MMA, together with the unreacted chitosan and NIPAAm, were mostly absorbed on the seeds and copolymerized to form the shell region of the particles. The chitosan and NIPAAm incorporated in the shell region would enhance the compatibility between core and shell region. In addition, they also form channels in the rigid shell to absorb or release drug and to prevent the disappearance of the thermoresponsive property when the rigid shell is expected to hinder the swelling of the core region. These channels formed by chitosan and NIPAAm in the shell region are also expected to let enzyme pass through the shell to degrade chitosan in the core when the endocytosis is caused by the ligand on the particle surface.

\subsubsection{Effect of MAA/MMA weight ratio}

From the conversion data in Table 2, the increase of MAA/MMA weight ratio accelerated the polymerization
Table 2

Conversion of poly(chitosan-NIPAAm/MAA-MMA) core-shell copolymerization for $1 \mathrm{~h}$ after MAA/MMA was fed into 2/10-3\% core latex with conversion of $82.02 \%$

\begin{tabular}{lll}
\hline Core type & MAA/MMA (w/w) & Conversion $(\%)$ \\
\hline $2 / 10-3 \%$ & $1 / 5$ & 85.63 \\
& $2 / 5$ & 86.79 \\
& $3 / 5$ & 88.34 \\
$2 / 10-3 \%$ & $0.5 / 2.5$ & 84.93 \\
& $1 / 5$ & 85.63 \\
& $2 / 10$ & 90.95 \\
\hline
\end{tabular}

rate. This could be due to the fact that the presence of MAA enhanced the copolymerization with the unreacted NIPAAm and chitosan, which thus increased the polymerization rate, when the hydrophilic oligomers or monomers (NIPAAm and chitosan) could not be absorbed or merged into the hydrophobic core particles (at the reaction temperature $50^{\circ} \mathrm{C}$ ) but could be dissolved in the MAA monomers to copolymerize the shell region.

\subsubsection{Effect of the concentration of shell monomers (MAA and MMA)}

From the conversion data in Table 2, the increase of the concentration of shell monomers accelerated the polymerization rate because more monomers were absorbed onto the seed particles to proceed the copolymerization reaction.

\subsection{Observation of latex particles}

Fig. 1a shows the SEM photograph of the poly(chitosanNIPAAm) core particles in the first stage of copolymerization, which exhibited higher hydrophilic and swelling properties at room temperature; therefore the boundary of the particles was indefinite. However, after the second stage reaction, the swelling of the core-shell particles was hindered by the rigid shell, so particles showed distinct boundaries, as shown in Figs. 1b-1d.

Fig. 2 shows the $\mathrm{COOH}$ groups distribution image of the copolymer particles. The uranyl acetate solution was used to selectively stain anionic (sulfate and carboxylic acid) groups in the particle cross sections. Under this observation, the anionic functional groups would show a darker image. It could be found that the copolymer particles thus synthesized exhibited a core-shell structure with the stained carboxylic acid groups (MAA) rich in the shell region. This result illustrates that the MAA and MMA monomers that were added in the second stage reaction formed the shell region successfully.

\subsection{Swelling measurements}

As the copolymerization reaction was carried out at $50^{\circ} \mathrm{C}$ which was higher than the LCST of PNIPAAm, the swelling of the final core-shell particles was hindered by the rigid shell even though the temperature was cooled to room tem- 

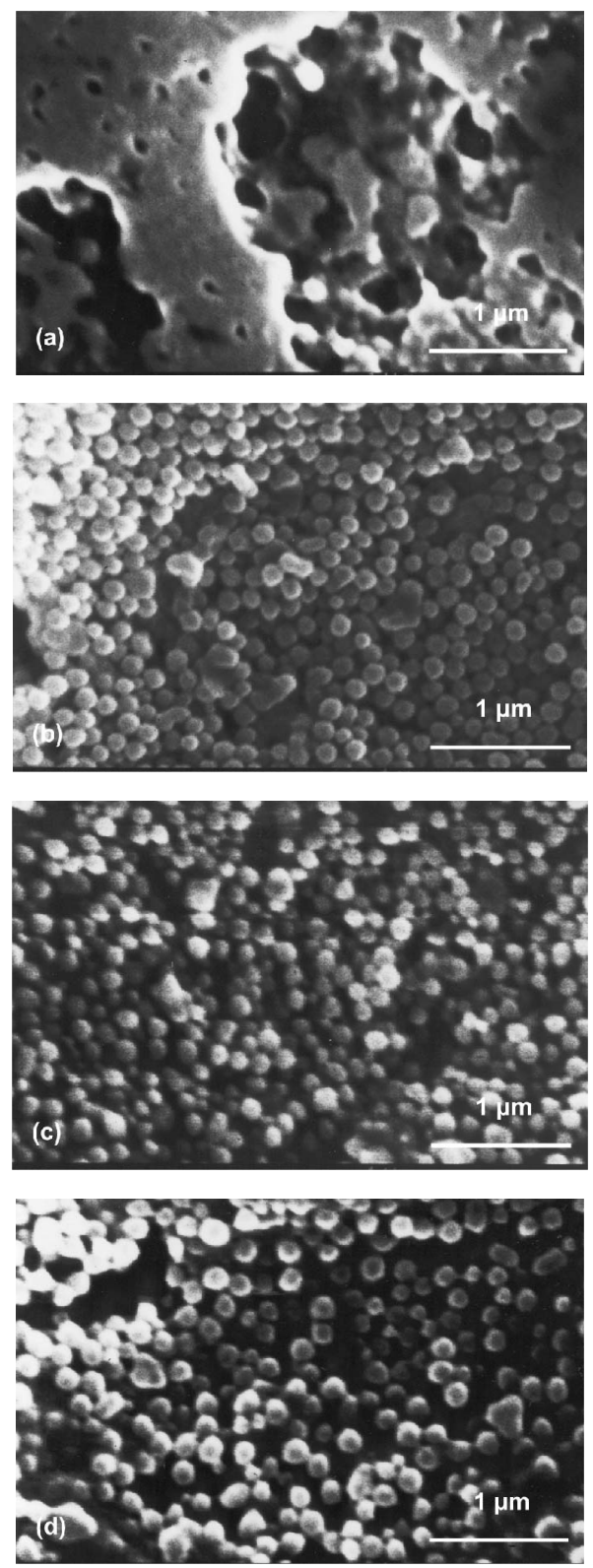

Fig. 1. SEM photographs of copolymer particles (a) 2/10-3\% (core particles without further copolymerization with MAA/MMA) and (b) 2/10-3\%-1/5, (c) $2 / 10-3 \%-3 / 5$, (d) 2/10-3\%-2/10 (the core-shell copolymer particles).

perature. So the swelling ratio and the thermoresponsivity of the core-shell type copolymer particles were not as good as those of poly(chitosan-NIPAAm) particles prepared in our previous study [27] although the phase transition temperature was still about $25-35^{\circ} \mathrm{C}$ shown in Fig. 3.

\subsubsection{Effect of $M A A / M M A$ weight ratio}

The swelling ratio of the sample increased with increasing the weight ratio of MAA/MMA in the temperature range of $20-40{ }^{\circ} \mathrm{C}$, due to the contribution of the hydrophilic property of MAA and the swelling ratio of these samples were too large to be measured accurately.

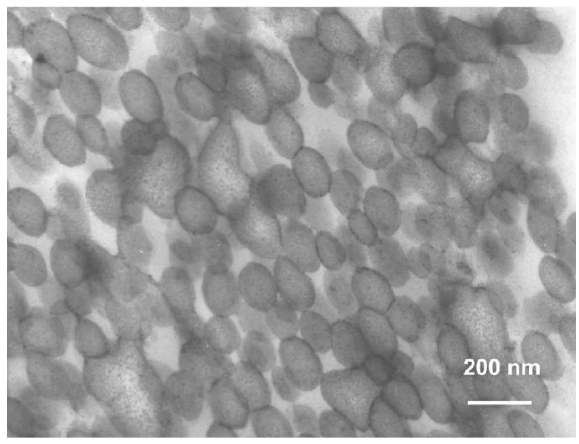

Fig. 2. Transmission electron micrographs of poly(chitosan-NIPAAm/ MAA-MMA) core-shell particles. Anionic sites were selectively stained using uranyl acetate to appear darker in this image.

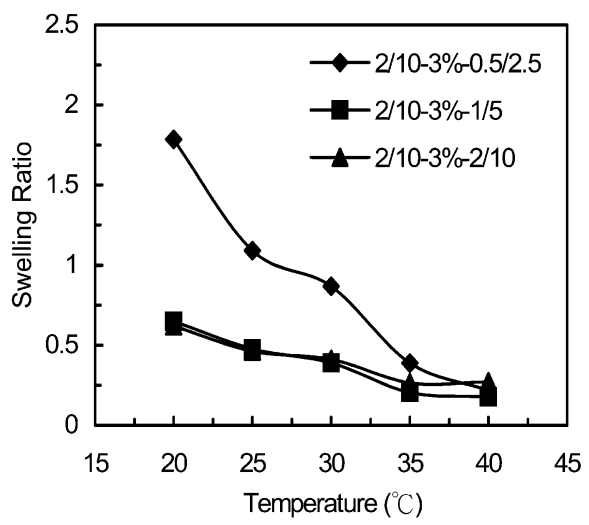

Fig. 3. The swelling ratio versus temperature for core-shell particles with different concentrations of shell monomers (MAA and MMA) in pH 7.4 phosphate buffer solutions.

\subsubsection{Effect of concentration of shell monomers}

Fig. 3 shows the swelling ratio versus temperature for the core-shell particles with various concentrations of shell monomers (MAA and MMA) in the phosphate buffer solution with $\mathrm{pH}$ 7.4. With a higher concentration of shell monomers, the shell of the particles was thicker, which seriously hindered the swelling of core-shell particles. In other words, if only small amounts of MAA and MMA were incorporated in copolymerization, as in sample $2 / 10-3 \%-0.5 / 2.5$, the particles formed with a thin shell exhibiting a higher swelling ratio and showing a thermal responsivity very similar to PNIPAAm.

The effect of $\mathrm{pH}$ value on the swelling ratio of poly(chitosan-NIPAAm/MAA-MMA) core-shell particles with various concentrations of shell monomers at $20^{\circ} \mathrm{C}$ was plotted in Fig. 4. In sample $2 / 10-3 \%-0.5 / 2.5$, the shell thickness was the smallest, so the swelling behavior was similar to PNIPAAm and exhibiting higher swelling ratio. In comparing the samples of $2 / 10-3 \%-1 / 5$ and $2 / 10-3 \%-2 / 10$, the sample with a high concentration of MAA and MMA showed a higher swelling ratio in $\mathrm{pH} 9$ buffer solution. It was mainly because the carboxylic acid group of MAA ionized to $\mathrm{COO}^{-}$. In addition, when the shell part of the particles was swollen in $\mathrm{pH} 9$ solution, swelling of the core part, PNIPAAm, also took place. Therefore, it could be concluded that 


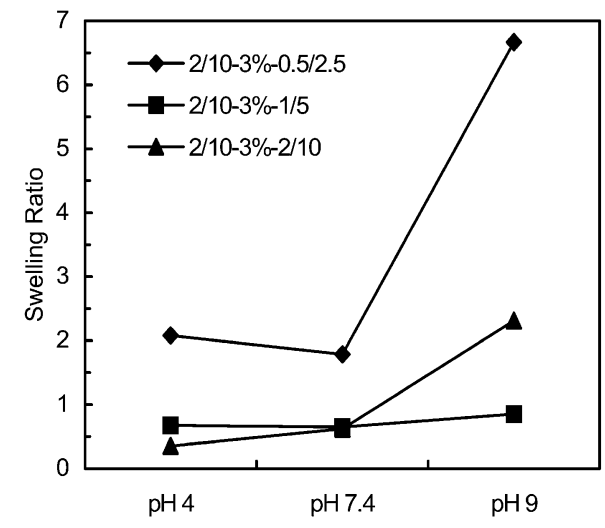

Fig. 4. The swelling ratio of the samples with different concentrations of shell monomers (MAA and MMA) in buffer solution with different $\mathrm{pH}$ values at $20^{\circ} \mathrm{C}$.

the swelling behavior in $\mathrm{pH} 9$ was influenced by the combination effect of the shell thickness, NIPAAm, and MAA.

Comparing the samples of $2 / 10-3 \%-1 / 5$ and $2 / 10-3 \%-$ $2 / 10$, the sample with a lower concentration of MAA and MMA showed a higher swelling ratio in $\mathrm{pH} 4$ buffer solution because of its smaller shell thickness would not hinder the swelling of NIPAAm in the core region seriously and the $\mathrm{NH}_{2}$ groups of chitosan would ionize to $\mathrm{NH}_{3}^{+}$. Therefore, it could be concluded that the swelling behavior was influenced by the combination effect of the shell thickness, NIPAAm, and chitosan.

\subsection{Zeta potential and average size of swollen particles}

The shell part of the core-shell particles was mainly the copolymers of MAA and MMA, so the zeta potential of particles was between -34 and $-40 \mathrm{mV}$ when the $\mathrm{pH}$ value was between 7.4 and 9 because the $\mathrm{COOH}$ group of MAA in the shell ionized to $\mathrm{COO}^{-}$(the $\mathrm{p} K_{a}$ value of PMAA was reported to be $4.3[29,30])$. Therefore, particles had more negative zeta potential when the weight ratio of MAA/MMA or the concentration of MAA and MMA increased. When the solution was changed to $\mathrm{pH} 4$, the $\mathrm{COOH}$ group of MAA in the shell was very stable in the $\mathrm{COOH}$ form, the $\mathrm{NH}_{3}^{+}$derived from $\mathrm{NH}_{2}$ of chitosan (the $\mathrm{p} K_{a}$ value of chitosan was reported to be $6.3[31,32]$ ) and the $\mathrm{SO}_{4}^{-}$derived from the initiator (ammonium persulfate) combined to determine the zeta potential of the particles. Consequently, the zeta potential of particles was very small in the $\mathrm{pH} 4$ buffer solution.

Figs. $5 \mathrm{a}$ and $5 \mathrm{~b}$ show the average diameter of swollen poly(chitosan-NIPAAm/MAA-MMA) core-shell particles with different weight ratios of MAA/MMA or different concentrations of MAA and MMA in buffer solution with different $\mathrm{pH}$ values at $25^{\circ} \mathrm{C}$. The average size of the swollen particles increased as the weight ratio of MAA/MMA or the concentration of shell monomers increased. This average diameter was the hydrodynamic diameter (swollen particle diameter) measured by laser light scattering, which was always larger than that observed by TEM or SEM. So the average diameter shown here could be influenced by not only
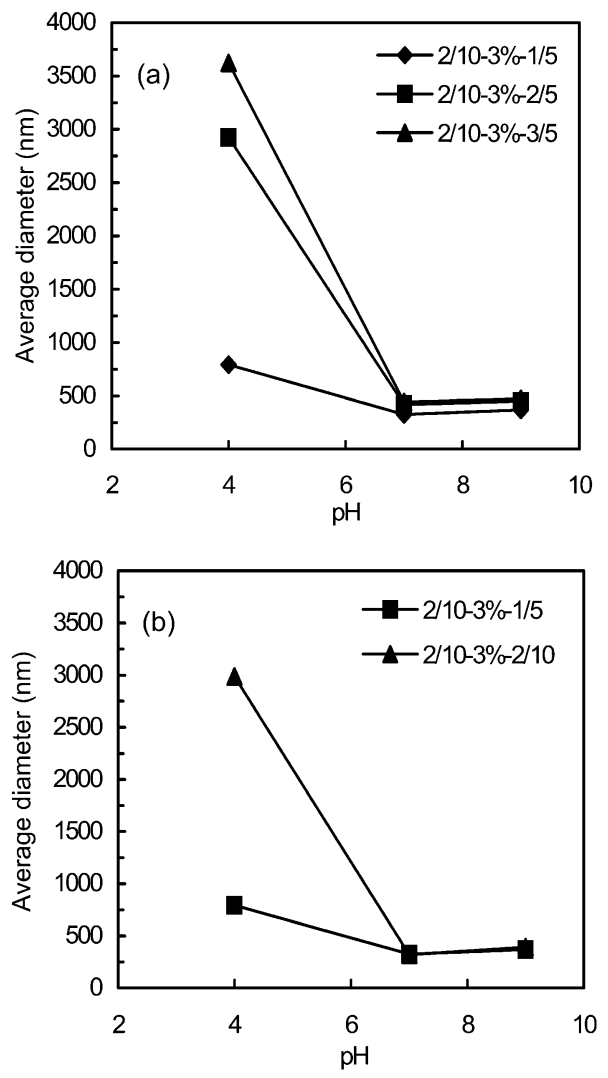

Fig. 5. Average diameter of swollen core-shell particles in buffer solution with different $\mathrm{pH}$ values at $25^{\circ} \mathrm{C}$ : (a) the effect of weight ratios of MAA/MMA; (b) the effect of concentrations of shell monomers (MAA and MMA).

the intrinsic size of particles but also the swelling and coagulation of particles. The particles in $\mathrm{pH} 9$ solution had a higher swelling ratio; the average diameter in $\mathrm{pH} 9$ was higher than that in $\mathrm{pH}$ 7.4. However, the average diameters measured in $\mathrm{pH} 4$ were found to be much larger than those in $\mathrm{pH} 7.4$ or 9 , although the swelling ratio of particles in $\mathrm{pH} 4$ was the smallest (Fig. 4). This was caused by the effect of particle coagulation since the zeta potential of particles was very small in $\mathrm{pH} 4$ solution as mentioned above.

\subsection{Specific surface area and surface functional group of particles}

The specific surface area of the core-shell particles with various weight ratios of MAA/MMA or various concentrations of shell monomers were measured and listed in Table 3. The MAA-rich sample, having a higher swelling ratio caused by the water absorption of MAA, formed a more porous structure after the freeze-drying process. Therefore, it was expected that the MAA-rich sample would have a larger specific surface area. But it should be noted that the samples with a much higher swelling ratio, $2 / 10$ $3 \%-3 / 5$ and $2 / 10-3 \%-0.5 / 2.5$, formed larger pores after the freeze-drying process than the other samples having a lower swelling ratio and smaller pores. Consequently, the trend of 
Table 3

Specific surface area and $\mathrm{COOH}$ groups of poly(chitosan-NIPAAm/MAAMMA) core-shell particles with various MAA/MMA weight ratios or various concentrations of shell monomers

\begin{tabular}{llll}
\hline Sample & $\begin{array}{l}\text { BET specific } \\
\text { surface area } \\
\left(\mathrm{m}^{2} / \mathrm{g}\right)\end{array}$ & $\begin{array}{l}\mathrm{COOH} \text { group } \\
\times 10^{-3} \\
(\mathrm{~mol} / \mathrm{g})\end{array}$ & $\begin{array}{l}\text { COOH group } \\
\times 10^{-4} \\
\left(\mathrm{~mol} / \mathrm{cm}^{2}\right)\end{array}$ \\
\hline $2 / 10-3 \%-1 / 5$ & 17.71 & 2.014 & 1.137 \\
$2 / 10-3 \%-2 / 5$ & 21.21 & 2.977 & 1.404 \\
$2 / 10-3 \%-3 / 5$ & 19.30 & 3.952 & 2.047 \\
$2 / 10-3 \%-0.5 / 2.5$ & 5.91 & - & - \\
$2 / 10-3 \%-1 / 5$ & 17.71 & 2.014 & 1.137 \\
$2 / 10-3 \%-2 / 10$ & 22.79 & 3.389 & 1.487 \\
\hline
\end{tabular}

specific surface area of particles with the weight ratio of MAA/MMA was to increase first and then decrease when the pore size effect became more important in the specific surface area measurements. And the sample of $2 / 10-3 \%-$ $0.5 / 2.5$ had the smallest specific surface area because of having the largest swelling ratio and a large pore structure.

Table 3 also shows the data of the amount of $\mathrm{COOH}$ groups on the surface of core-shell particles after the conductivity measurements of the $0.01 \mathrm{~N} \mathrm{NaOH}$ titration. The results indicate that the increase of the MAA/MMA weight ratio increased the $\mathrm{COOH}$ groups on the surface of particles significantly. The increase of $\mathrm{COOH}$ groups was not in proportion to the increase of MAA because the particle size and the specific surface area of each sample were not the same. Comparing the samples with different concentration of shell monomers, the one with thicker shell, 2/10-3\%-2/10, contained a higher amount of $\mathrm{COOH}$ groups per specific surface area of particles.

\subsection{Drug releasing estimation}

Caffeine was used as the model drug to carry out the drugreleasing test. The loading of caffeine into the latex particles was achieved by the swelling of copolymer particles in the pH 7.4 caffeine containing phosphate buffer solution; therefore the amount of caffeine loaded into the particles was in proportion to the equilibrium swelling ratio shown in Figs. 3 and 4. As expected, the drug might exist in both the particles and the solution. Since the particles were too small to be separated from the drug solution in both the drug loading and releasing processes, the caffeine-incorporated particles, together with the caffeine remaining in the solution (nonloaded caffeine), were molded into a tablet form in the drug release estimation. By this method, the released caffeine could be measured by UV spectrometer accurately when the interferences of latex particles were prevented. The drug-releasing behaviors of both nonloaded caffeine and loaded caffeine could be observed as seen in Fig. 6.

A reference copolymer tablet formed by blending copolymer particles with drug powders (not by way of swelling) was taken as the comparison. From Fig. 6, it was found that the blended caffeine was almost completely released
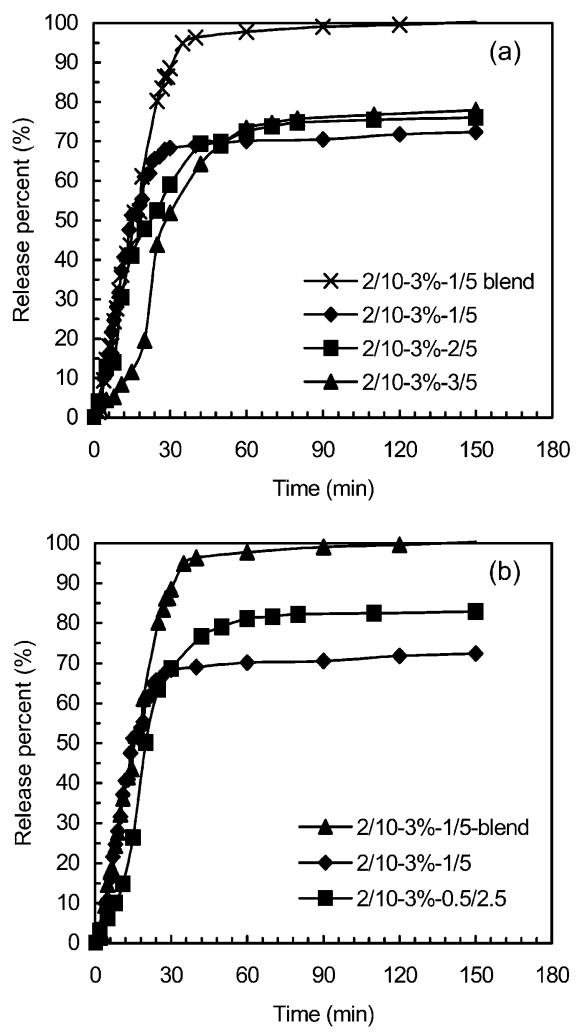

Fig. 6. The percentage of caffeine released from poly(chitosan-NIPAAm/ MAA-MMA) copolymer disks in the $\mathrm{pH} 7.4$ phosphate buffer solution at the temperature of $37^{\circ} \mathrm{C}$ : (a) the effect of weight ratios of MAA/MMA; (b) the effect of concentrations of shell monomers (MAA and MMA).

from the reference tablet at $37^{\circ} \mathrm{C}$ within $30 \mathrm{~min}$. In other samples loaded with caffeine by the swelling process, comparing with the release curve of the blend sample, the high proportion of drug loss $(70-80 \%)$ within 30 min was the amount of nonloaded drug, whereas the very slow release curve after $30 \mathrm{~min}$ (near zero release rate) indicated that the drug (20-30\%) that was really loaded into the particles (loaded drug) would be trapped and protected from release. From Fig. 6, although the efficiency of drug loading by the swelling process is only $20-30 \%$, the drug incorporated into the particles (loaded drug) could be increased by increasing the concentration of drug solution used in the swelling (loading) process.

For application, the nonloaded caffeine (about 70-80\%) should be removed from the sample. One way to do this is to dialyze the latex solution. Once the nonloaded drug is removed, the latex particles are expected to have a very low release rate. For targeting purposes, specific ligands should be conjugated on the surface of particles, and then these ligands and drug-containing latex particles will serve as vehicles for drug targeting applications. These particles could be used as oral medicines or injections in the form of powder, tablet, or latex solution according to the requirements of application.

The copolymer particles with a higher MAA/MMA weight ratio had a higher swelling ratio and thus absorbed 
more caffeine, but the caffeine incorporated into them could not be protected well and tended to diffuse into the solution because of its high swelling behavior. Therefore, it was found in Fig. 6a that the higher the weight ratio of MAA/MMA in the copolymer particles, the lower the amount of caffeine kept inside the particles in pH 7.4 PBS solution at $37^{\circ} \mathrm{C}$. The release rate of the MAA-rich sample was lower in the first $30 \mathrm{~min}$ because the caffeine was really absorbed inside the particles by swelling and then released into solution by diffusion. The same phenomenon was also found for thin-shell samples as seen in Fig. 6b. A lesser amount of caffeine was kept inside the particles of sample $2 / 10-3 \%-0.5 / 2.5$ after the release experiment. And the release rate of thin shell sample in the first $30 \mathrm{~min}$ was lower than that of the thicker shell sample.

\subsection{Protein conjugation}

In this work, the bovine serum albumin (BSA) was taken as model protein (ligand), which could be replaced with another protein according to the kind of wounded cells, to conjugate on the surface of copolymer particles by using EDC as a coupling agent to evaluate the protein conjugating ability and the potential of the core-shell copolymer particles being applied on the targeting drug carrier.

By utilizing the carbodiimide-mediated process, the $\mathrm{COOH}$ groups on the surface of core-shell particles combined with carbodiimide (EDC) to form an $o$-acrylisourea intermediate, then reacted with the $\mathrm{NH}_{2}$ group of BSA and yielded the corresponding amide bonding [33]. BSA was added to the latex of copolymer particles for $2 \mathrm{~h}$ before EDC. The formation of the electrostatic polymer-protein complexes proceeded, and then the condensation reaction between $\mathrm{COOH}$ and $\mathrm{NH}_{2}$ groups occurred after EDC was added.

For comparison, the same procedure was repeated without the addition of EDC. It was found that the amount of BSA remained in the solution was equal to the total amount of the added BSA; i.e., no BSA was adsorbed or entrapped in the particles during this procedure without the aid of EDC by using a centrifuge at $12,000 \mathrm{rpm}$ for $20 \mathrm{~min}$. In other words, the reduced amount of BSA in the solution is then confirmed to be chemically conjugated to the latex particles with the aid of EDC.

Fig. 7 shows the effect of temperature on the amounts of BSA conjugated on the surface of poly(chitosan-NIPAAm/ MAA-MMA) core-shell particles with various MAA/MMA weight ratios or various concentrations of shell monomers. The amounts of BSA conjugated on the surface of particles might be influenced by three factors: the size of swollen particles, the hydrophobic properties of particles, and the concentration of $\mathrm{COOH}$ groups on the particles. It is well known that the larger swollen particles reduces the steric hindrance of protein (BSA) conjugation, and the greater hydrophobic property of particles increases the adsorption of protein on the surface of particles. Therefore, the favored conditions for

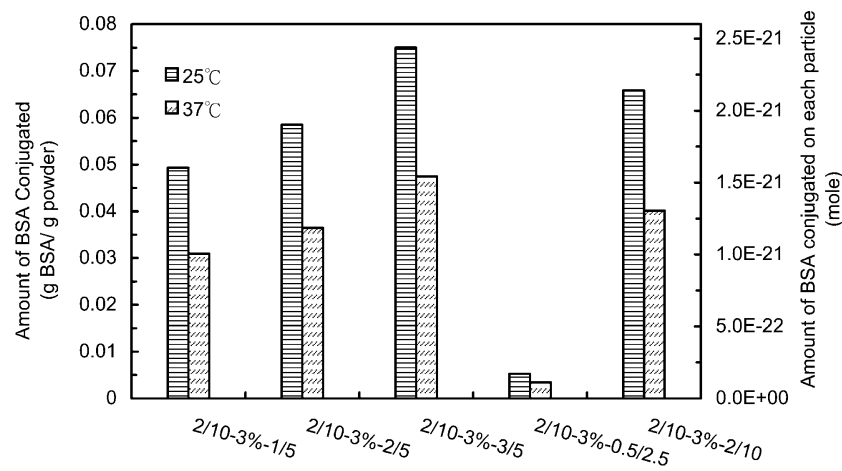

Fig. 7. The effect of temperature on the amount of BSA conjugated on the surfaces of core-shell particles with various MAA/MMA weight ratios or various concentrations of shell monomers (assuming the density of latex particles is $1 \mathrm{~g} / \mathrm{cm}^{3}$ and the average particle diameter is $160 \mathrm{~nm}$ according to the SEM observations).

protein conjugation are the larger swollen particle size, the greater hydrophobic property of the particles and the higher concentration of $\mathrm{COOH}$ groups on the particles. So at $37^{\circ} \mathrm{C}$ the amounts of BSA conjugated on particles increased when the MAA/MMA weight ratio or the concentration of shell monomers increased, because the concentration of $\mathrm{COOH}$ groups and the size of swollen particles increased. The very limited quantities of BSA conjugated on the sample of $2 / 10$ $3 \%-0.5 / 2.5$ resulted due to the strong hydrophilic properties and lower $\mathrm{COOH}$ groups of the sample. It was interesting to compare the results of the amounts of BSA conjugated on particles at 37 and $25^{\circ} \mathrm{C}$. The conjugation amount increased at $25^{\circ} \mathrm{C}$ because of the increase in particle size and the decrease in steric hindrance. In addition, the enhancement of BSA conjugation at $25^{\circ} \mathrm{C}$ compared to that at $37^{\circ} \mathrm{C}$ was increased as the MAA/MMA weight ratio increased because the swelling of samples was enhanced with the increasing of MAA/MMA weight ratio. The enhancement of the conjugation amount was limited for the sample of $2 / 10-3 \%-0.5 / 2.5$ with a thin shell, because the effect of its increasing hydrophilic property from 37 to $25^{\circ} \mathrm{C}$ was much stronger than in the other samples. It could be concluded that the thermoresponsive property of these core-shell particles is helpful to enhance the protein (ligand) conjugation at low temperature and these core-shell particles have the potential for drug targeting application.

\section{Conclusion}

In this study, the crosslinked poly(chitosan-NIPAAm/ MAA-MMA) thermal-sensitive and core-shell type copolymer particles were synthesized successfully. The relationships between the conversion, swelling, zeta potential, average diameter, specific surface area of particles, drug release and protein conjugation were studied by changing the weight ratio of MAA/MMA or the concentration of shell monomers. With an increase of the weight ratio of MAA/MMA or a decrease of the shell thickness of particles, the swelling ra- 
tio of the sample increased. In the $\mathrm{pH} 4$ buffer solution, the swelling behavior and the zeta potential of particles were influenced by the combination effect of shell thickness, NIPAAm, and chitosan. In pH 9 buffer solution, the swelling behavior and the zeta potential were influenced by the combination effect of shell thickness, NIPAAm, and MAA. In the drug release estimation, caffeine could be merged inside the copolymer particles by the swelling process and could be protected from releasing effectively in the phosphate buffer solution at $37^{\circ} \mathrm{C}$. In the experiments of protein conjugation, the amount of BSA conjugated on the surface of particles was influenced by the size of swollen particles, the hydrophobic properties of particles, and the surface $\mathrm{COOH}$ groups. In this study, the conjugation amount at either 37 or $25^{\circ} \mathrm{C}$ increased when the surface $\mathrm{COOH}$ groups and the size of particles increased, as in the MAA rich sample or the thick shell sample. The conjugation amount increased significantly at $25^{\circ} \mathrm{C}$ because of the increase in swollen particle size and the decrease in steric hindrance.

\section{References}

[1] M. Heskins, J.E. Guillet, E. James, J. Macromol. Sci. Chem. 8 (1968) 1441.

[2] Y.H. Bae, T. Okano, S.W. Kim, J. Polym. Sci. B Polym. Phys. 28 (1990) 923-936.

[3] T. Binkert, J. Oberreich, M. Meewes, R. Nyffenegger, J. Ricka, Macromolecules 24 (1991) 5806-5810.

[4] R.A. Stile, K.E. Healy, Biomacromolecules 3 (2002) 591-600.

[5] D. Schmaljohann, J. Oswald, B. Jorgensen, M. Nitschke, D. Beyerlein, C. Werner, Biomacromolecules 4 (2003) 1733-1739.

[6] A. Higuchi, A. Hamamura, Y. Shindo, H. Kitamura, B.O. Yoon, T. Mori, T. Uyama, A. Umezawa, Biomacromolecules 5 (2004) 17701774 .

[7] F.J. Xu, S.P. Zhong, L.Y.L. Yung, E.T. Kang, K.G. Neoh, Biomacromolecules 5 (2004) 2392-2403.

[8] M. Shibayama, T. Tanaka, C.C. Han, J. Chem. Phys. 97 (1992) 68426854.
[9] R. Yoshida, Y. Okuyama, K. Sakai, T. Okano, Y. Sakurai, J. Membrane Sci. 89 (1994) 267-277.

[10] C.S. Brazel, N.A. Peppas, Macromolecules 28 (1995) 8016-8020.

[11] M. Shibayama, S.Y. Mizutani, S. Nomura, Macromolecules 29 (1996) 2019-2024.

[12] H. Kawasaki, S. Sasaki, H. Maeda, J. Phys. Chem. B 101 (1997) 4184 4187.

[13] X.M. Zhang, Z.B. Hu, Y. Li, J. Appl. Polym. Sci. 63 (1997) 18511856.

[14] M.B. Huglin, Y. Liu, J.L. Velada, Polymer 38 (1997) 5785-5791.

[15] T.G. Park, Biomaterials 20 (1999) 517-521.

[16] A.S. Hoffman, A. Afressiabi, L.C. Dong, J. Control. Rel. 4 (1986) 213-222.

[17] K. Mukae, Y.H. Bae, T. Okano, S.W. Kim, Polym. J. 22 (1990) 250265.

[18] M. Wang, J. Qiang, Y. Fang, D. Hu, Y. Cui, X. Fu, J. Polym. Sci. A Polym. Chem. 38 (2000) 474-481.

[19] Y.H. Lim, D. Kim, D.S. Lee, J. Appl. Polym. Sci. 64 (1997) $2647-$ 2655.

[20] T. Okano, R. Yoshida, K. Sakai, Y. Sakurai, in: D. DeRossi, K. Kajiwara, Y. Osada, A. Yamauchi (Eds.), Polymer Gels-Fundamentals and Biomedical Applications, Plenum, New York, 1991, pp. 299-308.

[21] H. Yu, D.W. Grainger, Macromolecules 27 (1994) 4554-4560.

[22] L.C. Dong, A.S. Hoffman, J. Control. Rel. 15 (1991) 141-152.

[23] M.K. Yoo, Y.K. Sung, Y.M. Lee, C.S. Cho, Polymer 15 (2000) 57135719.

[24] S.Y. Kim, S.M. Cho, Y.M. Lee, S.J. Kim, J. Appl. Polym. Sci. 78 (2000) 1381-1391.

[25] W.F. Lee, Y.J. Chen, J. Appl. Polym. Sci. 82 (2001) 2487-2496.

[26] C.F. Lee, C.J. Wen, W.Y. Chiu, J. Polym. Sci. A Polym. Chem. 41 (2003) 2053-2063.

[27] C.F. Lee, C.J. Wen, C.L. Lin, W.Y. Chiu, J. Polym. Sci. A Polym. Chem. 42 (2004) 3029-3037.

[28] B.R. Saunders, B. Vincent, Adv. Colloid Interface Sci. 80 (1999) 1-25.

[29] C.R. Rajan, Y.S. Svinivas, S. Ponrathnam, K. Radhakrishnan, J. Polym. Sci. C Polym. Lett. 25 (2003) 73-77.

[30] E. Mouri, K. Matsumoto, H. Matsuoko, J. Appl. Crystallogr. 36 (2003) $722-726$.

[31] R.A.A. Muzzarelli, Chitin, Pergamon, New York, 1979, p. 103.

[32] H. Matsuyama, Y. Kitamura, Y. Naramura, J. Appl. Polym. Sci. 72 (1999) 397-404.

[33] A.S. Dilgimen, Z. Mustafaeva, M. Demchenko, T. Kaneko, Y. Osada, M. Mustafaev, Biomaterials 22 (2001) 2383-2392. 